\title{
Repellency of injured ascomata of Ciborinia camelliae and Spathularia flavida to fungivorous collembolans
}

\begin{abstract}
The repellency of injured ascomata of Ciborinia camelliae and Spathularia flavida was tested with the collembolan Ceratophysella denisana, a common mushroom feeder. Presentation of a test ascoma (intact or injured) at a distance to a collembolan that was feeding at a bait showed that significantly more collembolans moved away in response to the presentation of injured ascomata than intact ones for both species. These results suggest that the ascomata of these species showed repellency to the collembolan species as a response to injury. Odor is speculated to be one of the stimuli eliciting the behavioral responses observed.
\end{abstract}

Key words Ascomycetes · Assays · Ceratophysella denisana . Mushrooms

Ascomata are normally not attacked by collembolans, which are common consumers of basidiomata. Thus, these structures have been suspected to contain some protective agent.

A number of fungivorous animals injure fruit-bodies during feeding. Changes in bioactivity and/or chemical composition as a response to injury are known in basidiomata (Sterner et al. 1985; Pang et al. 1992; Wood et al. 1994, 2001; Stadler and Sterner 1998; Fäldt et al. 1999). In some cases, these changes are thought to serve as a chemical defense system against fungivores (Sterner et al. 1985; Stadler and Sterner 1998; Wood et al. 2001). However, no changes in bioactivity as a response to injury have yet been detected in ascomata (Stadler and Sterner 1998).

T. Nakamori $(\bowtie)$

Environmental Radiation Effects Research Group, National Institute of Radiological Sciences, 4-9-1 Anagawa, Inage-ku, Chiba 263-8555, Japan

Tel. +81-43-206-3255; Fax +81-43-206-3267

e-mail: taizo@fml.nirs.go.jp

A. Suzuki

Department of Biology, Faculty of Education, Chiba University,

Chiba, Japan
To test the possibility that ascomata show repellency, which is one of the defensive bioactivities, to collembolans in response to injury, we carried out assays involving observations of the behavioral responses of collembolans to intact or injured ascomata presented at a distance.

Ascomata of Ciborinia camelliae L. M. Kohn and Spathularia flavida Pres. were used for tests because we could obtain a sufficient number of ascomata of these species. Ascomata of C. camelliae were collected from Nango, Otsu, Shiga Prefecture, Japan, on April 5, 2005. Tests of this species were performed 4 days after collection (April 9, 2005). Ascomata of S. flavida were collected from Kawakami, Minamisaku, Nagano Prefecture, Japan on September 9,2004. Tests of this species were carried out 2 days after collection (September 11, 2004). Ascomata were stored at $5^{\circ}-10^{\circ} \mathrm{C}$ until use.

The collembolan species used for tests is Ceratophysella denisana Yosii (Hypogastrura denisana), a common fruitbody feeder in Japan (Nakamori and Suzuki 2005). Collembolans were obtained from the collecting site of ascomata of each species on March 3, 2005 for C. camelliae tests and on August 19, 2004 for S. flavida tests. They were fed with baker's yeast at $20^{\circ} \mathrm{C}$ until use.

To avoid injury of ascoma before the assay, fungal specimens were identified macroscopically, and identification of all the specimens of ascomata and collembolans was confirmed based on microscopic morphology at the end of the assay.

The test item was an intact ascoma or excised ascoma (the tip cut off with scissors, as shown in Fig. 1). The transection was made across the hymenium and the length was $5-10 \mathrm{~mm}$. In the case of injured ascoma, the cut edge was presented about $10 \mathrm{~s}$ after transecting.

The tests involved presentation of the ascoma (intact or injured) at a distance of $2 \mathrm{~mm}$ to collembolans that were feeding at a bait of baker's yeast on an agar disc (Fig. 2). The behavioral responses of the collembolans were then observed. A drop of water suspension of yeast was placed on the center of a $3 \%$ water agar disk $(3 \mathrm{~mm}$ in depth; $90 \mathrm{~mm}$ in diameter) in a petri dish, and excess water was removed with a piece of filter paper. One collembolan individual 


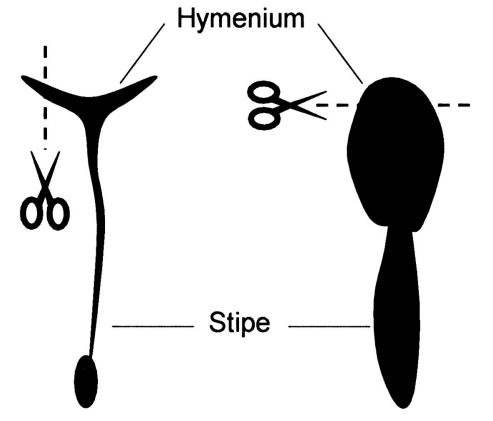

Ciborinia camelliae Spathularia flavida

Fig. 1. Transection method used for injuring ascomata. Ascomata are shown as a section in the right direction. Not to scale. Broken lines indicate transection

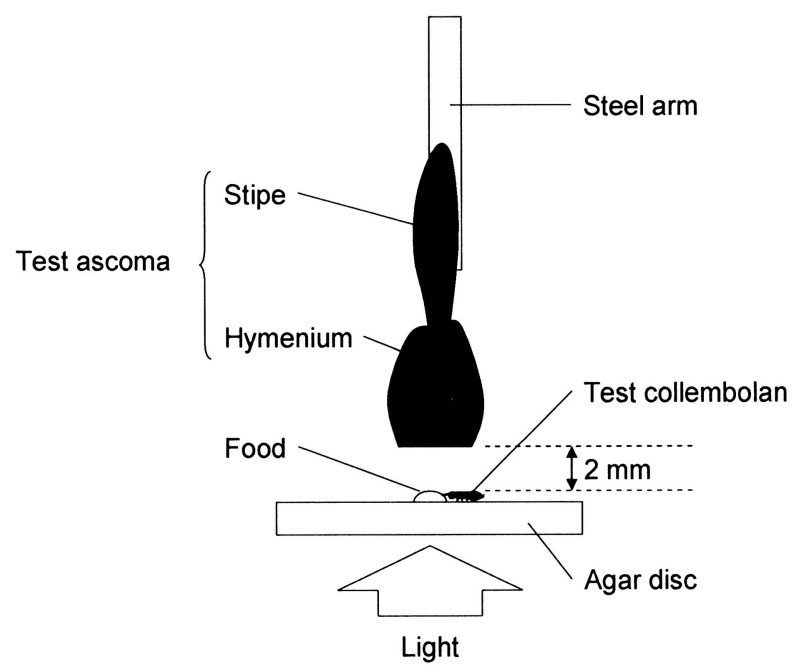

Fig. 2. Presentation method of the test ascoma. Ascoma is represented by injured Spathularia flavida. Not to scale

starved for a week was transferred onto the agar disc near the bait and then enclosed together with the bait with a cover vessel $(15 \mathrm{~mm}$ in diameter; $30 \mathrm{~mm}$ in height; transparent plastic). When the collembolan had settled to feed, the vessel was removed and the tip of a test ascoma was presented with a mechanical steel arm about $2 \mathrm{~mm}$ above the collembolan. The test ascoma was softly tied upside down to the steel arm by the stipe with Teflon tape. Distance of the collembolan from the bait was recorded 10 and $30 \mathrm{~s}$ after presentation of $C$. camelliae and S. flavida, respectively. When the collembolan moved outside the circle $(15 \mathrm{~mm}$ in diameter) around the bait, we regarded the collembolan as having shown a distributional response. In other cases, we considered that the collembolan did not show the response. To minimize electrostatic effects, both the stipe of ascoma and the agar disc were grounded. To exclude effects from the shadows of ascomata, an upwardly shining light was placed under the agar disc (semitransparent). The collembolan individuals used were not disturbed their feeding from three presentations of the steel arm immediately before the tests. The tests were carried out at $20^{\circ} \mathrm{C}$ in light.
All collembolan and ascomata specimens were used only once for either of two treatments, and data were treated as independent. Sample sizes were 12 and 15 replicates per treatment for C. camelliae and S. flavida, respectively. Differences in number of collembolan individuals showing the distributional response between treatments were tested by the Fisher exact probability test for each ascomycete species.

For both $C$. camelliae and $S$. flavida, significantly more collembolan individuals showed the distributional response to the presentation of injured ascomata (53\% and $42 \%$, respectively) than intact ones (both $0 \%$ ) (Fisher exact probability test, $P<0.05)$. All the collembolan individuals showing the distributional response walked away from the injured ascomata, except, in the case of S. flavida, one jumped.

Other behavioral responses were observed on collembolan individuals presented with injured ascomata of both the species. During presentation of an intact ascoma, none of the collembolan showed observable changes in behavior, whereas during presentation of an injured ascoma all individuals showed specific behavioral changes: all individuals stopped feeding at least once and moved their antennae in the air.

The bioactivity to $C$. denisana as a response to injury was clearly detected in ascomata of both $C$. camelliae and $S$. flavida. The bioactivity observed was thought to be repellency, because the presentation of injured ascomata elicited moving away from the ascomata and changes in distribution, and prevented feeding of the collembolan species.

Odor is speculated to be one of the stimuli of the bioactivity observed, because the collembolans responded at a distance and moved their antennae. Chemoreceptors are most probably located at the antennae of collembolans (Altner and Altner 1985). Odor is one of the important cues affecting collembolan behavior (Bengtsson et al. 1988, 1991; Hedlund et al. 1995; Messer et al. 1999; Nilsson and Bengtsson 2004a,b; Pfander and Zettel 2004).

Odor is a cue both attracting and repelling collembolans. Attractant odors in food are known (Bengtosson et al. 1988, 1991; Hedlund et al. 1995), whereas only a few repellent odors in food have previously been identified. Interestingly, the ascomycetes Tuber melanosporum Vitt. contains 1,3dimethoxybenzene, known as an alarm substance in the collembolan Neanura muscorum Willem (Messer et al. 1999, and a reference therein).

Changes in the bioactivity of odor as a response to injury are known in basidiomata. Wood et al. (2001) showed that the volatile 1-octen-3-ol from Clitopilus prunulus (Scop.: Fr.) Kummer acts as a banana slug antifeedant. Injured basidiomata of the bracket fungi Fomitopsis pinicola (Fr.) P. Karst. and Fomes fometarius (L.) J.J. Kickx showed increased release of oct-1-en-3-ol, known as an insect attractant (Fäldt et al. 1999).

The bioactivity observed in the present study might also be induced by biting of $C$. denisana, and this might repel the collembolans. Therefore, the bioactivity is speculated to serve to protect the ascomata of C. camelliae and S. flavida from the collembolan species, as is thought to occur in basidiomata (Sterner et al. 1985; Stadler and Sterner 1998; Wood et al. 2001). 
Acknowledgments We thank Dr. M. Kakishima, Institute of Agriculture and Forestry, University of Tsukuba, Dr. K. Uchida, Dr. Y. Kuroda, and other staff of the Agricultural and Forestry Research Centre of Tsukuba University, for allowing access to the Kawakami University Forest of Tsukuba University.

\section{References}

Altner H, Altner I (1985) Multicellular antennal sensilla containing a sensory cell with a short dendrite and dense-core granules in the insect, Hypogastrura socialis (Collembola): intermolt and molting stages. Cell Tissue Res 241:119-128

Bengtsson G, Erlandsson A, Rundgren S (1988) Fungal odour attracts soil Collembola. Soil Biol Biochem 20:25-30

Bengtsson G, Hedlund K, Rundgren S (1991) Selective odor perception in the soil Collembola Onychiurus armatus. J Chem Ecol 17:2113-2125

Fäldt J, Jonsell M, Nordlander G, Borg-Karlson A-K (1999) Volatiles of bracket fungi Fomitopsis pinicola and Fomes fomentarius and their functions as insect attractants. J Chem Ecol 25:567-590

Hedlund K, Bengtsson G, Rundgren S (1995) Fungal odour discrimination in two sympatric species of fungivorous collembolans. Funct Ecol 9:869-875
Messer C, Dettner K, Schulz S, Francke W (1999) Phenolic compounds in Neanura muscorm (Collembola, Neanuridae) and the role of 1,3dimethoxybenzene as an alarm substance. Pedobiologia 43:174-182

Nakamori T, Suzuki A (2005) Spore-breaking capabilities of collembolans and their feeding habit within sporocarps. Pedobiologia 49:261267

Nilsson E, Bengtsson G (2004a) Death odour changes movement pattern of a Collembola. Oikos 104:509-517

Nilsson E, Bengtsson G (2004b) Endogenous free fatty acids repel and attract Collembola. J Chem Ecol 30:1431-1443

Pang Z, Sterner O, Anke H (1992) (8E)-10-Hydroxydec-8-enoic acid: its isolation from injured fruit-bodies of Cantharellus tubaeformis and synthetic preparation. Acta Chem Scand 46:301-303

Pfander I, Zettel J (2004) Chemical communication in Ceratophysella sigillata (Collembola: Hypogastruridae): intraspecific reaction to alarm substances. Pedobiologia 48:575-580

Stadler M, Sterner O (1998) Production of bioactive secondary metabolites in the fruit bodies of macrofungi as a response to injury. Phytochemistry 49:1013-1019

Sterner O, Bergman R, Kihlberg J, Wickberg B (1985) The sesquiterpens of Lactarius vellereus and their role in a proposed chemical defense system. J Nat Prod 48:279-288

Wood WF, Brandes ML, Watson RL, Jones RL, Largent DL (1994) trans-2-Nonenal, the cucumber odor of mushrooms. Mycologia 86: $561-563$

Wood WF, Archer CL, Largent DL (2001) 1-Octen-3-ol, a banana slug antifeedant from mushroom. Biochem Syst Ecol 28:89-90 